\title{
Pre competition anxiety among athletes
}

\author{
Sumudu V. Rajasinghe ${ }^{1}$, Dilini V. Vipulaguna ${ }^{2}$ \\ ${ }^{1}$ Sports Medicine Unit, T.H. Anuradhapura. \\ ${ }^{2}$ Faculty of Medicine, Colombo.
}

\section{Introduction}

Mental preparation is one of the most important part of an individual's sports performance. Anxiety caused by impending competition will lead to problems in sleep and reduction in performance. Most players do not realize this and they blame for other factors for their poor performance.

\section{Objectives}

To assess the level of anxiety and psychological factors that can negatively affect the performance of the players during the competition.

\section{Method}

50 players aged 16-40 years were assessed by an interviewer administered questionnaire including SCAT (Sport competition anxiety test) during the North central provincial sports meet 2011.

\section{Results}

Most players $(54 \%, \mathrm{n}=27)$ were athletes and $34(68 \%)$ were involved in individual events. Sixteen players were (32\%) between 21-25 years of age and 74\% $(n=37)$ were males. Majority $(72 \%, n=36)$ had average SCAT score but 26\% $(n=13)$ had high score. $52 \%(n=26)$ players had problems related to sleep before their competition and players involved in individual events experienced more sleep problems and high SCAT score.64\% $(n=32)$ were not aware about the coping strategies like relaxation and $96 \%(n=48)$ of players never used it.

\section{Discussion}

Pre competition anxiety will adversely affect performance. Identification of anxiety among players is utmost important and coping strategies like relaxation should be taught to players as well as coaches to control pre competition anxiety. 